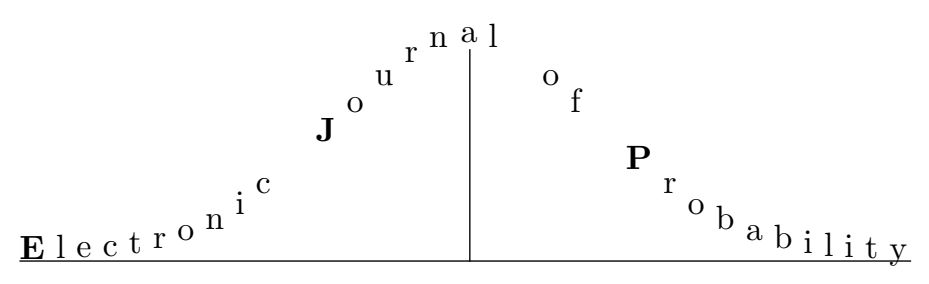

Vol. 13 (2008), Paper no. 2, pages 5-25.

Journal URL

http://www.math.washington.edu/ ejpecp/

\title{
Local Energy Statistics in Directed Polymers.
}

\author{
Irina Kurkova *
}

\begin{abstract}
Recently, Bauke and Mertens conjectured that the local statistics of energies in random spin systems with discrete spin space should, in most circumstances, be the same as in the random energy model. We show that this conjecture holds true as well for directed polymers in random environment. We also show that, under certain conditions, this conjecture holds for directed polymers even if energy levels that grow moderately with the volume of the system are considered .
\end{abstract}

Key words: Simple random walk on $\mathbf{Z}^{d}$, Gaussian random variables, directed polymers, Poisson point process.

AMS 2000 Subject Classification: Primary 60K35.

Submitted to EJP on February 14, 2007, final version accepted December 18, 2007.

${ }^{*}$ Laboratoire de Probabilités et Modèles Aléatoires, Université Paris 6, B.C. 188; 4, place Jussieu, 75252 Paris Cedex 05, France. E-mail : kourkova@ccr.jussieu.fr 


\section{Motivation and historical overview}

Recently, Bauke and Mertens have proposed in [2] a new and original look at disordered spin systems. This point of view consists of studying the micro-canonical scenario, contrary to the canonical formalism, that has become the favorite tool to treat models of statistical mechanics. More precisely, they analyze the statistics of spin configurations whose energy is very close to a given value. In discrete spin systems, for a given system size, the Hamiltonian will take on a finite number of random values, and generally (at least, if the disorder is continuous) a given value $E$ is attained with probability 0 . One may, however, ask : How close to $E$ the best approximant is when the system size grows and, more generally, what the distribution of the energies that come closest to $E$ is ? Finally, how the values of the corresponding configurations are distributed in configuration space?

The original motivation for this viewpoint came from a reformulation of a problem in combinatorial optimization, the number partitioning problem (this is the problem of partitioning $N$ (random) numbers into two subsets such that their sums in these subsets are as close as possible) in terms of a spin system Hamiltonian $[1 ; 20 ; 21]$. Mertens conjecture stated in these papers

has been proven to be correct in [5] (see also [8]), and generalized in [9] for the partitioning into $k>2$ subsets.

Some time later, Bauke and Mertens generalized this conjecture in the following sense : let $\left(H_{N}(\sigma)\right)_{\sigma \in \Sigma_{N}}$ be the Hamiltonian of any disordered spin system with discrete spins $\left(\Sigma_{N}\right.$ being the configuration space) and continuously distributed couplings, let $E$ be any given number, then the distribution of the close to optimal approximants of the level $\sqrt{N} E$ is asymptotically (when the volume of the system $N$ grows to infinity) the same as if the energies $H_{N}(\sigma)$ are replaced by independent Gaussian random variables with the same mean and variance as $H_{N}(\sigma)$ (that is the same as for Derrida's Random Energy spin glass Model [13], that is why it is called the REM conjecture).

What this distribution for independent Gaussian random variables is ? Let $X$ be a standard Gaussian random variable, let $\delta_{N} \rightarrow 0$ as $N \rightarrow \infty, E \in \mathbf{R}, b>0$. Then it is easy to compute that

$$
\mathrm{P}\left(X \in\left[E-\delta_{N} b, E+\delta_{N} b\right]\right)=\left(2 \delta_{N} b\right) \sqrt{1 /(2 \pi)} e^{-E^{2} / 2}(1+o(1)) \quad N \rightarrow \infty .
$$

Let now $\left(X_{\sigma}\right)_{s \in \Sigma_{N}}$ be $\left|\Sigma_{N}\right|$ independent standard Gaussian random variables. Since they are independent, the number of them that are in the interval $\left[E-\delta_{N} b, E+\delta_{N} b\right]$ has a Binomial distribution with parameters $\left(2 \delta_{N} b\right) \sqrt{1 /(2 \pi)} e^{-E^{2} / 2}(1+o(1))$ and $\left|\Sigma_{N}\right|$. If we put

$$
\delta_{N}=\left|\Sigma_{N}\right|^{-1} \sqrt{2 \pi}(1 / 2) e^{E^{2} / 2},
$$

by a well known theorem of the course of elementary Probability, this random number converges in law to the Poisson distribution with parameter $b$ as $N \rightarrow \infty$. More generally, the point process

$$
\sum_{\sigma \in \Sigma_{N}} \delta_{\left\{\delta_{N}^{-1} N^{-1 / 2}\left|\sqrt{N} X_{\sigma}-\sqrt{N} E\right|\right\}}
$$

converges, as $N \rightarrow \infty$, to the Poisson point process in $\mathbf{R}_{+}$whose intensity measure is the Lebesgue measure. 
So, Bauke and Mertens conjecture states that for the Hamiltonian $\left(H_{N}(\sigma)\right)_{\sigma \in \Sigma_{N}}$ of any disordered spin system and for a suitable normalization $C(N, E)$ the sequence of point processes

$$
\sum_{\sigma \in \Sigma_{N}} \delta_{\left\{C(N, E)\left|H_{N}(\sigma)-\sqrt{N} E\right|\right\}}
$$

converges, as $N \rightarrow \infty$, to the Poisson point process in $\mathbf{R}_{+}$whose intensity measure is the Lebesgue measure. In other words, the best approximant to $\sqrt{N} E$ is at distance $C^{-1}(N, E) W$, where $W$ is an exponential random variable of mean 1 . More generally, the $k$ th best approximant to $\sqrt{N} E$ is at distance $C^{-1}(N, E)\left(W_{1}+\cdots+W_{k}\right)$, where $W_{1}, \ldots, W_{k}$ are independent exponential random variables of mean $1, k=1,2 \ldots$ It appears rather surprising that such a result holds in great generality. Indeed, it is well known that the correlations of the random variables are strong enough to modify e.g. the maxima of the Hamiltonian. This conjecture has been proven in [10] for a rather large class of disordered spin systems including short range lattice spin systems as well as mean-field spin glasses, like $p$-spin Sherringthon-Kirkpatrick (SK) models with Hamiltonian $H_{N}(\sigma)=N^{1 / 2-p / 2} \sum_{1 \leq i_{1}, \ldots, i_{p} \leq N} \sigma_{i_{1}} \cdots \sigma_{i_{p}} J_{i_{1}, \ldots, i_{p}}$ where $J_{i_{1}, \ldots, i_{p}}$ are independent standard Gaussian random variables, $p \geq 1$. See also [6] for the detailed study of the case $p=1$.

Two questions naturally pose themselves. (i) Consider instead of $E, N$-dependent energy levels, say, $E_{N}=\operatorname{const} N^{\alpha}$. How fast can we allow $E_{N}$ to grow with $N \rightarrow \infty$ for the same behavior (i.e. convergence to the standard Poisson point process under a suitable normalization) to hold ? (ii) What type of behavior can we expect once $E_{N}$ grows faster than this value ?

The first question (i) has been investigated for Gaussian disordered spin systems in [10]. It turned out that for short range lattice spin systems on $\mathbf{Z}^{d}$ this convergence is still true up to $\alpha<1 / 4$. For mean-field spin glasses, like $p$-spin SK models with Hamiltonian $H_{N}(\sigma)=$ $N^{1 / 2-p / 2} \sum_{i_{1}, \ldots, i_{p}} \sigma_{i_{1}} \cdots \sigma_{i_{p}} J_{i_{1}, \ldots, i_{p}}$ mentioned above, this conjecture holds true up to $\alpha<1 / 4$ for $p=1$ and up to $\alpha<1 / 2$ for $p \geq 2$. It has been proven in [7] that the conjecture fails at $\alpha=1 / 4$ for $p=1$ and $\alpha=1 / 2$ for $p=2$. The paper [7] extends also these results for non-Gaussian mean-field 1-spin SK models with $\alpha>0$.

The second question (ii), that is the local behavior beyond the critical value of $\alpha$, where Bauke and Mertens conjecture fails, has been investigated for Derrida's Generalized Random Energy Models ([14]) in [11].

Finally, the paper [3] introduces a new REM conjecture, where the range of energies involved is not reduced to a small window. The authors prove that for large class of random Hamiltonians the point process of properly normalized energies restricted to a sparse enough random subset of spin configuration space converges to the same point process as for the Random Energy Model, i.e. Poisson point process with intensity measure $\pi^{-1 / 2} e^{-t \sqrt{2 \ln 2}} d t$.

In this paper we prove Bauke and Merten's conjecture on the local behavior of energies not for disordered spin systems but for directed polymers in random environment. To our knowledge, this is the first study of this conjecture out of its original domain of disordered spin systems. Let $\left(\left\{w_{n}\right\}_{n \geq 0}, P\right)$ be a simple random walk on the $d$-dimensional lattice $\mathbf{Z}^{d}$. More precisely, we let $\Omega$ be the path space $\Omega=\left\{\omega=\left(\omega_{n}\right)_{n \geq 0} ; \omega_{n} \in \mathbf{Z}^{d}, n \geq 0\right\}, \mathcal{F}$ be the cylindrical $\sigma$-field on $\Omega$ and for all $n \geq 0, \omega_{n}: \omega \rightarrow \omega_{n}$ be the projection map. We consider the unique probability measure $P$ on $(\Omega, \mathcal{F})$ such that $\omega_{1}-\omega_{0}, \ldots, \omega_{n}-\omega_{n-1}$ are independent and

$$
P\left(\omega_{0}=0\right)=1, \quad P\left(\omega_{n}-\omega_{n-1}= \pm \delta_{j}\right)=(2 d)^{-1}, \quad j=1, \ldots, d,
$$


where $\delta_{j}=\left(\delta_{k j}\right)_{k=1}^{d}$ is the $j$ th vector of the canonical basis of $\mathbf{Z}^{d}$. We will denote by $S_{N}=$ $\left\{\omega^{N}=\left(i, \omega_{i}\right)_{i=0}^{N}\right\} \quad\left(\left(i, \omega_{i}\right) \in \mathbf{N} \times \mathbf{Z}^{d}\right)$ the space of paths of length $N$. The polymer chain is represented as a graph $\left\{\left(i, \omega_{i}\right)\right\}_{i=1}^{N}$ in $\mathbf{N} \times \mathbf{Z}^{d}$ where each point stands for the position of the $i$ th monomer. Let $\left\{\eta(n, x): n \in \mathbf{N}, x \in \mathbf{Z}^{d}\right\}$ be a sequence of independent identically distributed random variables of zero mean and variance 1 on a probability space $(H, \mathcal{G}, \mathrm{P})$. They describe the random environment, i.e. impurities at sites $(n, x)$. The polymer is attracted by large positive values of the environment and repelled by large negative ones. We define the energy of the chain $\omega^{N}=\left(i, \omega_{i}\right)_{i=0}^{N}$ as

$$
\eta\left(\omega^{N}\right)=N^{-1 / 2} \sum_{i=1}^{N} \eta\left(i, \omega_{i}\right)
$$

The typical shape of the polymer is then given by the one that maximizes the value (1). This model first appeared in physics literature [17] to modelize the phase boundary of Ising model subject to random impurities and its first mathematical study was undertaken by Imbrie and Spencer [18] and Bolthausen [4]. It relates - and sometimes can be mapped - to a number of interesting models of growing random surfaces (directed percolation, ballistic deposition, polynuclear growth, low temperature Ising models) and non equilibrium dynamics (totally asymmetric simple exclusion, population dynamics in random environment), see [19]. An increasing interest for these models is showing up in the mathematical community, see [12] for a survey of the main results and references therein. All these very interesting results are found in the frame of the traditional approach of statistical mechanics to the study of the energy spectrum (1) : on takes a parameter $\beta>0$ prescribing how strongly the polymer path $\omega$ interacts with the medium and then analyzes the distribution of the random polymer measure on the path space

$$
\mu_{N}(d \omega)=\frac{1}{Z_{N}} \exp \left(\beta \sum_{i=1}^{N} \eta\left(i, \omega_{i}\right)\right) P(d \omega)
$$

where $Z_{N}$ is the normalizing constant. For example, one investigates the asymptotic behavior of the quantities $\mu_{N}\left[\left|\omega_{N}\right|^{2}\right]$ or $\sup _{x \in \mathbf{Z}^{d}} \mu_{N-1}\left\{\omega_{N}=x\right\}$ as $N \rightarrow \infty$ depending on $\beta$ and dimension $d$. In this paper we propose a different point of view on the distribution of the energy spectrum (1), namely to study its local behavior compare to a given level $E_{N}$, which is constant or growing with $N \rightarrow \infty$.

\section{Results}

Our first theorem extends Bauke and Merens conjecture for directed polymers.

Theorem 1. Let $\eta(n, x),\left\{\eta(n, x): n \in \mathbf{N}, x \in \mathbf{Z}^{d}\right\}$, be the i.i.d. random variables of the third moment finite and with the Fourier transform $\phi(t)$ such that $|\phi(t)|=O\left(|t|^{-1}\right),|t| \rightarrow \infty$. Let $E_{N}=c \in \mathbf{R}$ and let

$$
\delta_{N}=\sqrt{\pi / 2} e^{c^{2} / 2}\left((2 d)^{N}\right)^{-1} .
$$

Then the point process

$$
\sum_{\omega^{N} \in S_{N}} \delta_{\left\{\delta_{N}^{-1}\left|\eta\left(\omega^{N}\right)-E_{N}\right|\right\}}
$$


converges weakly as $N \uparrow \infty$ to the Poisson point process $\mathcal{P}$ on $\mathbf{R}_{+}$whose intensity measure is the Lebesgue measure. Moreover, for any $\epsilon>0$ and any $b \in \mathbf{R}_{+}$

$$
\begin{gathered}
\mathrm{P}\left(\forall N_{0} \exists N \geq N_{0}, \exists \omega^{N, 1}, \omega^{N, 2}: \operatorname{cov}\left(\eta\left(\omega^{N, 1}\right), \eta\left(\omega^{N, 2}\right)\right)>\epsilon:\right. \\
\left.\left|\eta\left(\omega^{N, 1}\right)-E_{N}\right| \leq\left|\eta\left(\omega^{N, 2}\right)-E_{N}\right| \leq \delta_{N} b\right)=0 .
\end{gathered}
$$

The claim (1) of this theorem concerns the spatial distribution of paths that give the best approximants of $E_{N}$. It says that for any $b>0$, any $l \geq 2$ and any $l$-tuple of paths $\omega^{1}, \ldots, \omega^{l}$ where the approximations of $E_{N}$ with $\delta_{N} b$-precision are realized, $\omega^{1}, \ldots, \omega^{l}$ are asymptotically at the maximal distance between each other in the sense of $\operatorname{dist}\left(\omega^{N, 1}, \omega^{N, 2}\right)=1-N^{-1} \#\left\{i: \omega_{i}^{N, 1}=\right.$ $\left.\omega_{i}^{N, 2}\right\}$. This implies that for any $k>0, k$ paths $\omega^{1}, \ldots, \omega^{k}$ where the best approximations of $E_{N}$ are realized, are asymptotically at the maximal distance between each other.

The decay assumption on the Fourier transform is not optimal in this theorem, we believe that it can be weaken but we did not try to optimize it. Nevertheless, some condition of this type is needed, the result can not be extended for discrete distributions where the number of possible values the Hamiltonian takes on would be finite.

The next two theorems prove Bauke and Mertens conjecture for directed polymers in Gaussian environment for growing levels $E_{N}=c N^{\alpha}$. We are able to prove that this conjecture holds true for $\alpha<1 / 4$ for polymers in dimension $d=1$ et and $\alpha<1 / 2$ in dimension $d \geq 2$. We leave this investigation open for non-Gaussian environments.

The values $\alpha=1 / 4$ for $d=1$ and $\alpha=1 / 2$ for $d \geq 2$ are likely to be the true critical values. Note that these are the same as for Gaussian SK-spin glass models for $p=1$ and $p=2$ respectively according to [7], and likely for $p \geq 3$ as well.

Theorem 2. Let $\eta(n, x),\left\{\eta(n, x): n \in \mathbf{N}, x \in \mathbf{Z}^{d}\right\}$, be independent standard Gaussian random variables. Let $d=1$. Let $E_{N}=c N^{\alpha}$ with $c \in \mathbf{R}, \alpha \in[0,1 / 4[$ and

$$
\delta_{N}=\sqrt{\pi / 2} e^{E_{N}^{2} / 2}\left(2^{N}\right)^{-1} .
$$

Then the point process

$$
\sum_{\omega^{N} \in S_{N}} \delta_{\left\{\delta_{N}^{-1}\left|\eta\left(\omega^{N}\right)-E_{N}\right|\right\}}
$$

converges weakly as $N \uparrow \infty$ to the Poisson point process $\mathcal{P}$ on $\mathbf{R}_{+}$whose intensity measure is the Lebesgue measure. Moreover, for any $\epsilon>0$ and any $b \in \mathbf{R}_{+}$

$$
\begin{gathered}
\mathrm{P}\left(\forall N_{0} \exists N \geq N_{0}, \exists \omega^{N, 1}, \omega^{N, 2}: \operatorname{cov}\left(\eta\left(\omega^{N, 1}\right), \eta\left(\omega^{N, 2}\right)\right)>\epsilon:\right. \\
\left.\left|\eta\left(\omega^{N, 1}\right)-E_{N}\right| \leq\left|\eta\left(\omega^{N, 2}\right)-E_{N}\right| \leq \delta_{N} b\right)=0 .
\end{gathered}
$$

Theorem 3. Let $\eta(n, x),\left\{\eta(n, x): n \in \mathbf{N}, x \in \mathbf{Z}^{d}\right\}$ be independent standard Gaussian random variables. Let $d \geq 2$. Let $E_{N}=c N^{\alpha}$ with $c \in \mathbf{R}, \alpha \in[0,1 / 2[$ and

$$
\delta_{N}=\sqrt{\pi / 2} e^{E_{N}^{2} / 2}\left((2 d)^{N}\right)^{-1} .
$$

Then the point process

$$
\sum_{\omega^{N} \in S_{N}} \delta_{\left\{\delta_{N}^{-1}\left|\eta\left(\omega^{N}\right)-E_{N}\right|\right\}}
$$


converges weakly as $N \uparrow \infty$ to the Poisson point process $\mathcal{P}$ on $\mathbf{R}_{+}$whose intensity measure is the Lebesgue measure. Moreover, for any $\epsilon>0$ and any $b \in \mathbf{R}_{+}$

$$
\begin{gathered}
\mathrm{P}\left(\forall N_{0} \exists N \geq N_{0}, \exists \omega^{N, 1}, \omega^{N, 2}: \operatorname{cov}\left(\eta\left(\omega^{N, 1}\right), \eta\left(\omega^{N, 2}\right)\right)>\epsilon:\right. \\
\left.\left|\eta\left(\omega^{N, 1}\right)-E_{N}\right| \leq\left|\eta\left(\omega^{N, 2}\right)-E_{N}\right| \leq \delta_{N} b\right)=0 .
\end{gathered}
$$

Acknowledgements. The author thanks Francis Comets for introducing him to the area of directed polymers. He also thanks Stephan Mertens and Anton Bovier for attracting his attention to the local behavior of disordered spin systems and interesting discussions.

\section{Proofs of the theorems.}

Our approach is based on the following sufficient condition of convergence to the Poisson point process. It has been proven in a somewhat more general form in [9].

Theorem 4. Let $V_{i, M} \geq 0, i \in \mathbf{N}$, be a family of non-negative random variables satisfying the following assumptions : for any $l \in \mathbf{N}$ and all sets of constants $b_{j}>0, j=1, \ldots, l$

$$
\lim _{M \rightarrow \infty} \sum_{\left(i_{1}, \ldots, i_{l}\right) \in\{1, \ldots, M\}} \mathrm{P}\left(\forall_{j=1}^{l} V_{i_{j}, M}<b_{j}\right)=\prod_{j=1}^{l} b_{j}
$$

where the sum is taken over all possible sequences of different indices $\left(i_{1}, \ldots, i_{l}\right)$. Then the point process

$$
\sum_{i=1}^{M} \delta_{\left\{V_{i, M}\right\}}
$$

on $\mathbf{R}_{+}$converges weakly in distribution as $M \rightarrow \infty$ to the Poisson point process $\mathcal{P}$ on $\mathbf{R}_{+}$whose intensity measure is the Lebesgue measure.

Hence, to prove the convergence of point processes (3), (6) and (9), we just have to verify the hypothesis of Theorem 4 for $V_{i, M}$ given by $\delta_{N}^{-1}\left|\eta\left(\omega^{N, i}\right)-E_{N}\right|$, i.e. we must show that

$$
\sum_{\left(\omega^{N, 1}, \ldots, \omega^{N, l}\right) \in S_{N}^{l}} \mathrm{P}\left(\forall_{i=1}^{l}:\left|\eta\left(\omega^{N, i}\right)-E_{N}\right|<b_{i} \delta_{N}\right) \rightarrow b_{1} \cdots b_{l}
$$

where the sum is taken over all sets of different paths $\left(\omega^{N, 1}, \ldots, \omega^{N, l}\right)$.

Informal proof of Theorem 1. Before proceeding with rigorous proofs let us give some informal arguments supporting Theorem 1 .

The random variables $\eta\left(\omega^{N, i}\right), i=1, \ldots, l$, are the sums of independent identically distributed random variables with zero mean. The $l \times l$ covariance matrix $B_{N}\left(\omega^{N, 1}, \ldots, \omega^{N, l}\right)$ of $l$ random variables $\eta\left(\omega^{N, i}\right), i=1, \ldots, l$, has 1 on the diagonal and the covariances cov $\left(\eta\left(\omega^{N, i}\right), \eta\left(\omega^{N, j}\right)\right)=$ $N^{-1} \#\left\{m: \omega_{m}^{N, i}=\omega_{m}^{N, j}\right\} \equiv b_{i, j}(N)$ for $i \neq j, i, j \in\{1, \ldots, l\}$.

The number of sets $\left(\omega^{N, 1}, \ldots, \omega^{N, l}\right)$ with $b_{i, j}(N)=o(1)(o(1)$ should be chosen of an appropriate order) for all pairs $i \neq j, i, j=1, \ldots, l$, as $N \rightarrow \infty$, is $(2 d)^{N l}(1-\gamma(N))$ as $N \rightarrow \infty$ where $\gamma(N)$ is 
exponentially small in $N$. For all such sets $\left(\omega^{N, 1}, \ldots, \omega^{N, i}\right)$, by the local Central Limit Theorem, the random variables $\eta\left(\omega^{N, i}\right), i=1, \ldots, l$, should behave asymptotically as Gaussian random variables with covariances $b_{i, j}(N)=o(1)$ and the determinant of the covariance matrix $1+o(1)$. Therefore, the probability that these random variables belong to $\left[-\delta_{N} b_{i}+c, \delta_{N} b_{i}+c\right]$ respectively for $i=1, \ldots, l$, equals

$$
\left(2 \delta_{N} b_{1}\right) \cdots\left(2 \delta_{N} b_{l}\right)(\sqrt{2 \pi})^{-l} e^{-c^{2} l / 2}=b_{1} \cdots b_{l} 2^{-N l}(1+o(1)) .
$$

Since the number of such sets $\left(\omega^{N, 1}, \ldots, \omega^{N, l}\right)$ is $(2 d)^{N l}(1+o(1))$, the sum (11) over them converges to $b_{1} \cdots b_{l}$.

Let us turn to the remaining tiny part of $S_{N}^{l}$ where $\left(\omega^{N, 1}, \ldots, \omega^{N, l}\right)$ are such that the covariances $b_{i, j}(N) \neq o(1)$ with $o(1)$ of an appropriate order for some $i \neq j, i, j=1, \ldots, l, N \rightarrow \infty$. The number of such sets is exponentially smaller than $(2 d)^{N l}$. Here two possibilities should be considered differently.

The first one is when the covariance matrix is non-degenerate. Then, invoking again the Central Limit Theorem, the probabilities $\mathrm{P}(\cdot)$ in (11) in this case are not greater than

$$
\left(\operatorname{det} B_{N}\left(\omega^{N, 1}, \ldots, \omega^{N, l}\right)\right)^{-1 / 2}\left(2 \delta_{N} b_{1}\right) \cdots\left(2 \delta_{N} b_{l}\right)(\sqrt{2 \pi})^{-l} .
$$

From the definition of the covariances of $\eta\left(\omega^{N, i}\right)$, det $B_{N}\left(\omega^{N, 1}, \ldots, \omega^{N, l}\right)$ is a finite polynomial in the variables $1 / N$. Therefore the probabilities $\mathrm{P}(\cdot)$ in $(11)$ are bounded by $(2 d)^{-N l}$ up to a polynomial term, while the number of sets $\left(\omega^{N, 1}, \ldots, \omega^{N, l}\right)$ such that $b_{i, j}(N) \neq o(1)$ some $i \neq j, i, j=1, \ldots, l$, is exponentially smaller than $(2 d)^{N l}$. Therefore the sum (11) over such sets $\left(\omega^{N, 1}, \ldots, \omega^{N, l}\right)$ converges to zero exponentially fast.

Let now $\left(\omega^{N, 1}, \ldots, \omega^{N, l}\right)$ be such that $B_{N}\left(\omega^{N, 1}, \ldots, \omega^{N, l}\right)$ is degenerate of the rank $r<l$. Then, without loss of generality, we may assume that $\eta\left(\omega^{N, 1}\right), \ldots, \eta\left(\omega^{N, r}\right)$ are linearly independent, while $\eta\left(\omega^{N, r+1}\right), \ldots, \eta\left(\omega^{N, l}\right)$ are their linear combinations. Then the probabilities $\mathrm{P}(\cdot)$ in (11) are bounded by the probabilities that only $\eta\left(\omega^{N, 1}\right), \ldots, \eta\left(\omega^{N, r}\right)$ belong to the corresponding intervals, which are at most $2^{-N r}$ up to a polynomial term as previously. Moreover, we will show that for no one $m=0,1, \ldots, N, \omega_{m}^{N, 1}, \ldots, \omega_{m}^{N, r}$ can not be all different. Otherwise, each of $\omega^{N, r+1}, \ldots, \omega^{N, l}$ would coincide with one of $\omega^{N, 1}, \ldots, \omega^{N, r}$, which is impossible since the sum (11) is taken over sets of different(!) paths. This implies that the number of such sets $\left(\omega^{N, 1}, \ldots, \omega^{N, r}\right)$ is exponentially smaller than $2^{N r}$. Furthermore, the number of possibilities to complete each of these sets by $\omega^{N, r+1}, \ldots, \omega^{N, l}$ such that $\eta\left(\omega^{N, r+1}\right), \ldots, \eta\left(\omega^{N, l}\right)$ are linear combinations of $\eta\left(\omega^{N, 1}\right), \ldots, \eta\left(\omega^{N, r}\right)$ is $N$-independent. Thus the number of sets $\left(\omega^{N, 1}, \ldots, \omega^{N, l}\right)$ in this case being exponentially smaller than $2^{N r}$, and the probabilities being $2^{-N r}$ up to a polynomial term, the corresponding sum (11) converges to zero. This completes the informal proof of (3) in Theorem 1 .

We now give rigorous proofs. We start with proofs of Theorems 2 and 3 in Gaussian environment and give the proof of Theorem 1 after that.

Proof of Theorem 2. For $\theta \in] 0,1 / 2[$ let us denote by

$$
\mathcal{R}_{N, l}^{\theta}=\left\{\left(\omega^{N, 1}, \ldots, \omega^{N, l}\right): \operatorname{cov}\left(\eta\left(\omega^{N, i}\right), \eta\left(\omega^{N, j}\right)\right) \leq N^{\theta-1 / 2}, \forall i, j=1, \ldots, l, i \neq j\right\} .
$$

Step 1. As a first preparatory step, we need to estimate the cardinality of $S_{N}^{l} \backslash \mathcal{R}_{N, l}^{\theta}$, i.e. to show (14). Let us first note that for any two paths $\omega^{N, 1}, \omega^{N, 2} \in S_{N}$

$$
\operatorname{cov}\left(\eta\left(\omega^{N, 1}\right), \eta\left(\omega^{N, 2}\right)\right)=s / N
$$


if and only if

$$
\#\left\{m:\left(\omega_{m}^{1}, m\right)=\left(\omega_{m}^{2}, m\right)\right\}=s,
$$

i.e. the number of moments of time within the period $[0, N]$ when the trajectories $\omega^{N, 1}$ and $\omega^{N, 2}$ are at the same point of the space $\mathbf{Z}$ equals $s$. But due to the symmetry of the simple random walk

$$
\begin{aligned}
& \#\left\{\omega^{N, 1}, \omega^{N, 2}: \#\left\{m \in[0, \ldots, N]: \omega_{m}^{1}-\omega_{m}^{2}=0\right\}=s\right\} \\
= & \#\left\{\omega^{N, 1}, \omega^{N, 2}: \#\left\{m \in[0, \ldots, N]: \omega_{m}^{1}+\omega_{m}^{2}=0\right\}=s\right\} .
\end{aligned}
$$

Taking into account the fact that the random walk starting from 0 can not visit 0 at odd moments of time, we obtain that (3) equals

$$
\#\left\{\omega^{2 N}: \#\left\{m \in[0, \ldots, 2 N]: \omega_{m}=0\right\}=s\right\} .
$$

This last number is well-known for the simple random walk on $\mathbf{Z}$ : it equals $2^{2 N} \times 2^{s-2 N}\left(\begin{array}{c}2 N-s \\ N\end{array}\right)$ (see e.g. [16], Volume 1, Chapter 3, Section 10, exercise 10) which is, by Stirling's formula, when $\left.s=\left[N^{1 / 2+\theta}\right], \theta \in\right] 0,1 / 2\left[\right.$, equivalent to $2^{2 N}(\pi N)^{-1 / 2} e^{-s^{2} /(4 N)}=2^{2 N}(\pi N)^{-1 / 2} e^{-N^{2 \theta} / 4}$ as $N \rightarrow \infty$. Finally, we obtain that for all $N \geq 0$ the number (3) it is not greater than $2^{2 N} e^{-h N^{2 \theta}}$ with some constant $h>0$. It follows that for all $N>0$

$$
\begin{aligned}
& \left|S_{N}^{l} \backslash \mathcal{R}_{N, l}^{\theta}\right| \\
& \leq(l(l-1) / 2) 2^{N(l-2)} \#\left\{\omega^{N, 1}, \omega^{N, 2}: \#\left\{m \in[0, \ldots, N]: \omega_{m}^{1}-\omega_{m}^{2}=0\right\} \geq N^{1 / 2+\theta}\right\} \\
& \leq 2^{N l} C N \exp \left(-h N^{2 \theta}\right)
\end{aligned}
$$

where $C>0, h>0$ are some constants.

Step 2. The second preparatory step is the estimation (3) and (18) of the probabilities in the sum (11). Let $B_{N}\left(\omega^{N, 1}, \ldots, \omega^{N, l}\right)$ be the covariance matrix of the random variables $\eta\left(\omega^{N, i}\right)$ for $i=1, \ldots, l$. Then, if $B_{N}\left(\omega^{N, 1}, \ldots, \omega^{N, l}\right)$ is non-degenerate,

$$
\mathrm{P}\left(\forall_{i=1}^{l}:\left|\eta\left(\omega^{N, i}\right)-E_{N}\right|<b_{i} \delta_{N}\right)=\int_{C\left(\vec{E}_{N}\right)} \frac{e^{-\left(\vec{z} B_{N}^{-1}\left(\omega^{N, 1}, \ldots, \omega^{N, l}\right) \vec{z}\right) / 2}}{(2 \pi)^{l / 2} \sqrt{\operatorname{det} B_{N}\left(\omega^{N, 1}, \ldots, \omega^{N, l}\right)}} d \vec{z}
$$

where

$$
C\left(\vec{E}_{N}\right)=\left\{\vec{z}=\left(z_{1}, \ldots, z_{l}\right):\left|z_{i}-E_{N}\right| \leq \delta_{N} b_{i}, \forall i=1, \ldots, l\right\} .
$$

Let $\theta \in] 0,1 / 2\left[\right.$. Since $\delta_{N}$ is exponentially small in $N$, we see that uniformly for $\left(\omega^{N, 1}, \ldots, \omega^{N, l}\right) \in$ $\mathcal{R}_{N, l}^{\theta}$, the probability (15) equals

$$
\begin{aligned}
& \left(2 \delta_{N} / \sqrt{2 \pi}\right)^{l}\left(b_{1} \cdots b_{l}\right) e^{-\left(\vec{E}_{N} B_{N}^{-1}\left(\omega^{N, 1}, \ldots, \omega^{N, l}\right) \vec{E}_{N}\right) / 2}(1+o(1)) \\
& =\left(2 \delta_{N} / \sqrt{2 \pi}\right)^{l}\left(b_{1} \cdots b_{l}\right) e^{-\left\|\vec{E}_{N}\right\|^{2}\left(1+O\left(N^{\theta-1 / 2}\right)\right) / 2}(1+o(1))
\end{aligned}
$$

where we denoted by $\vec{E}_{N}$ the vector $\left(E_{N}, \ldots, E_{N}\right)$.

We will also need a more rough estimate of the probability (15) out of the set $\mathcal{R}_{N, l}^{\theta}$. Let now the matrix $B_{N}\left(\omega^{N, 1}, \ldots, \omega^{N, l}\right)$ be of the rank $r \leq l$. Then, if $r<l$, there are $r$ paths among 
$\omega^{N, 1}, \ldots, \omega^{N, l}$ such that corresponding $r$ random variables $\eta\left(\omega^{N, i}\right)$ form the basis. Without loss of generality we may assume that these are $\omega^{N, 1}, \ldots, \omega^{N, r}$. Then the matrix $B_{N}\left(\omega^{N, 1}, \ldots, \omega^{N, r}\right)$ is non-degenerate and $\eta\left(\omega^{N, r+1}\right), \ldots, \eta\left(\omega^{N, l}\right)$ are linear combinations of $\eta\left(\omega^{N, 1}\right), \ldots, \eta\left(\omega^{N, r}\right)$. We may now estimate from above the probabilities (11) by the probabilities $\mathrm{P}\left(\forall_{i=1}^{r}:\left|\eta\left(\omega^{N, i}\right)-E_{N}\right|<\right.$ $\left.b_{i} \delta_{N}\right)$ that can be expressed in terms of the $r$-dimensional integrals like (15). Consequently, in this case

$$
\mathrm{P}\left(\forall_{i=1}^{l}:\left|\eta\left(\omega^{N, i}\right)-E_{N}\right|<b_{i} \delta_{N}\right) \leq \frac{\left(2 \delta_{N} / \sqrt{2 \pi}\right)^{r} b_{1} \cdots b_{r}}{\sqrt{\operatorname{det} B_{N}\left(\omega^{N, 1}, \ldots, \omega^{N, r}\right)}} .
$$

From the definition of the matrix elements, one sees that $\operatorname{det} B_{N}\left(\omega^{N, 1}, \ldots, \omega^{N, l}\right)$ is a finite polynomial in the variables $1 / N$. Hence, if the rank of $B\left(\omega^{N, 1}, \ldots, \omega^{N, r}\right)$ equals $r$, we have for all $N>0$

$$
\mathrm{P}\left(\forall_{i=1}^{l}:\left|\eta\left(\omega^{N, i}\right)-E_{N}\right|<b_{i} \delta_{N}\right) \leq 2^{-N r} e^{c^{2} r N^{2 \alpha} / 2} N^{k(r)}
$$

for some $k(r)>0$.

Step 3. Armed with (14), (3) and (18), we now proceed with the proof of the theorem.

For given $\alpha \in] 0,1 / 4\left[\right.$, let us choose first $\left.\theta_{0} \in\right] 0,1 / 4[$ such that

$$
2 \alpha-1 / 2+\theta_{0}<0
$$

Next, let us choose $\theta_{1}>\theta_{0}$ such that

$$
2 \alpha-1 / 2+\theta_{1}<2 \theta_{0}
$$

then $\theta_{2}>\theta_{1}$ such that

$$
2 \alpha-1 / 2+\theta_{2}<2 \theta_{1}
$$

etc. After $i-1$ steps we choose $\theta_{i}>\theta_{i-1}$ such that

$$
2 \alpha-1 / 2+\theta_{i}<2 \theta_{i-1}
$$

Let us take e.g. $\theta_{i}=(i+1) \theta_{0}$. We stop the procedure at $n=\left[\alpha / \theta_{0}\right]$ th step, that is

$$
n=\min \left\{i \geq 0: \alpha<\theta_{i}\right\} .
$$

Note that $\theta_{n-1} \leq \alpha<1 / 4$, and then $\theta_{n}=\theta_{n-1}+\theta_{0}<1 / 2$.

We will prove that the sum (11) over $\mathcal{R}_{N, l}^{\theta_{0}}$ converges to $b_{1} \cdots b_{l}$, while those over $\mathcal{R}_{N, l}^{\theta_{i}} \backslash \mathcal{R}_{N, l}^{\theta_{i-1}}$ for $i=1,2, \ldots, n$ and the one over $S_{N}^{l} \backslash \mathcal{R}_{N, l}^{\theta_{n}}$ converge to zero.

By (3), each term of the sum (11) over $\mathcal{R}_{N, l}^{\theta_{0}}$ equals

$$
\left(2 \delta_{N} / \sqrt{2 \pi}\right)^{l}\left(b_{1} \cdots b_{l}\right) e^{-\left\|\vec{E}_{N}\right\|^{2}\left(1+O\left(N^{\theta_{0}-1 / 2}\right)\right) / 2}(1+o(1)) .
$$

Here $e^{\left\|\vec{E}_{N}\right\|^{2} \times O\left(N^{\theta_{0}-1 / 2}\right)}=1+o(1)$ by the choice (19) of $\theta_{0}$. Then, by the definition of $\delta_{N}(5)$, each term of the sum (11) over $\mathcal{R}_{N, l}^{\theta_{0}}$ is

$$
\left(b_{1} \cdots b_{l}\right) 2^{-N l}(1+o(1))
$$


uniformly for $\left(\omega^{N, 1}, \ldots, \omega^{N, l}\right) \in \mathcal{R}_{N, l}^{\theta_{0}}$. The number of terms in this sum is $\left|\mathcal{R}_{N, l}^{\theta_{0}}\right|$, that is $2^{N l}(1+o(1))$ by (14). Hence, the sum (11) over $\mathcal{R}_{N, l}^{\theta_{0}}$ converges to $b_{1} \cdots b_{l}$.

Let us consider the sum over $\mathcal{R}_{N, l}^{\theta_{i}} \backslash \mathcal{R}_{N, l}^{\theta_{i-1}}$ for $i=1,2, \ldots, n$. Each term in this sum equals

$$
\left(2 \delta_{N} / \sqrt{2 \pi}\right)^{l}\left(b_{1} \cdots b_{l}\right) e^{-\left\|\vec{E}_{N}\right\|^{2}\left(1+O\left(N^{\theta_{i}-1 / 2}\right) / 2\right.}(1+o(1))
$$

uniformly for $\left(\omega^{N, 1}, \ldots, \omega^{N, l}\right) \in \mathcal{R}_{N, l}^{\theta_{i}}$. Then, by the definition of $\delta_{N}$ (5), it is bounded by $2^{-N l} C_{i} e^{h_{i} N^{2 \alpha-1 / 2+\theta_{i}}}$ with some constants $C_{i}, h_{i}>0$. The number of terms in this sum is not greater than $\left|S_{N}^{l} \backslash \mathcal{R}_{N, l}^{\theta_{i-1}}\right|$ which is bounded due to (14) by $C N 2^{N l} \exp \left(-h N^{2 \theta_{i-1}}\right)$. Then by the choice of $\theta_{i}(22)$ this sum converges to zero exponentially fast.

Let us now treat the sum over $S_{N}^{l} \backslash \mathcal{R}_{N, l}^{\theta_{n}}$. Let us first study the sum over $\left(\omega^{N, 1}, \ldots, \omega^{N, l}\right)$ such that the matrix $B_{N}\left(\omega^{N, 1}, \ldots, \omega^{N, l}\right)$ is non-degenerate. By (18) each term in this sum is bounded by $2^{-N l} e^{c^{2} l N^{2 \alpha} / 2} N^{k(l)}$ for some $k(l)>0$. The number of terms in this sum is bounded by $C N 2^{N l} \exp \left(-h N^{2 \theta_{n}}\right)$ by (14). Since $\alpha<\theta_{n}$ by (23), this sum converges to zero exponentially fast.

Let us finally turn to the sum over $\left(\omega^{N, 1}, \ldots, \omega^{N, l}\right)$ such that the matrix $B\left(\omega^{N, 1}, \ldots, \omega^{N, l}\right)$ is degenerate of the rank $r<l$. By (18) each term in this sum is bounded by

$$
2^{-N r} e^{c^{2} r N^{2 \alpha} / 2} N^{k(r)}
$$

for some $k(r)>0$.

There are $r$ paths among $\omega^{N, 1}, \ldots, \omega^{N, l}$ such that corresponding $\eta\left(\omega^{N, i}\right)$ form the basis. Without loss of generality we may assume that these are $\omega^{N, 1}, \ldots, \omega^{N, r}$. Note that $\omega^{N, 1}, \ldots, \omega^{N, r}$ are such that it can not be for no one $m \in[0, \ldots, N]$ that $\omega_{m}^{1}, \ldots, \omega_{m}^{r}$ are all different. In fact, assume that $\omega_{m}^{1}, \ldots, \omega_{m}^{r}$ are all different. Then $\eta\left(m, \omega_{m}^{1}\right), \ldots, \eta\left(m, \omega_{m}^{r}\right)$ are independent identically distributed random variables and $\eta\left(m, \omega_{m}^{r+1}\right)=\mu_{1} \eta\left(m, \omega_{m}^{1}\right)+\cdots+\mu_{r} \eta\left(m, \omega_{m}^{r}\right)$. If $\omega_{m}^{r+1}$ is different from all $\omega_{m}^{1}, \ldots, \omega_{m}^{r}$, then $\eta\left(m, \omega_{m}^{r+1}\right)$ is independent from all of $\eta\left(m, \omega_{m}^{1}\right), \ldots, \eta\left(m, \omega_{m}^{r}\right)$, then the linear coefficients, being the covariances of $\eta\left(m, \omega_{m}^{r+1}\right)$ with $\eta\left(m, \omega_{m}^{1}\right), \ldots, \eta\left(m, \omega_{m}^{r}\right)$, are $\mu_{1}=$ $\cdots=\mu_{r}=0$. So, $\eta\left(\omega^{N, r+1}\right)$ can not be a non-trivial linear combination of $\eta\left(\omega^{N, 1}\right), \ldots, \eta\left(\omega^{N, r}\right)$. If $\omega_{m}^{r+1}$ equals one of $\omega_{m}^{1}, \ldots, \omega_{m}^{r}$, say $\omega_{m}^{i}$, then again by computing the covariances of $\eta\left(m, \omega_{m}^{r+1}\right)$ with $\eta\left(m, \omega_{m}^{1}\right), \ldots, \eta\left(m, \omega_{m}^{r}\right)$, we get $\mu_{i}=1, \mu_{j}=0$ for $j=1, \ldots, i-1, i+1, \ldots, r$. Consequently, $\eta\left(\omega_{k}^{i}\right)=\eta\left(\omega_{k}^{r+1}\right)$ for all $k=1, \ldots, N$, so that $\omega^{N, i}=\omega^{N, r+1}$. But this is impossible since the sum (11) is taken over different paths $\omega^{N, 1}, \ldots, \omega^{N, l}$. Thus the sum is taken only over paths $\omega^{N, 1}, \ldots, \omega^{N, r}$ where at each moment of time at least two of them are at the same place.

The number of such sets of $r$ different paths is exponentially smaller than $2^{N r}$ : there exists $p>0$ such that is does not exceed $2^{N r} e^{-p N}$. (In fact, consider $r$ independent simple random walks on $\mathbf{Z}$ that at a given moment of time occupy any $k<r$ different points of $\mathbf{Z}$. Then with probability not less than $(1 / 2)^{r}$, at the next moment of time, they occupy at least $k+1$ different points. Then with probability not less than $\left((1 / 2)^{r}\right)^{r}$ at least once during $r$ next moments of time they will occupy $r$ different points. So, the number of sets of different $r$ paths that at each moment of time during $[0, N]$ occupy at most $r-1$ different points is not greater than $2^{N r}\left(1-\left(1 / 2^{r}\right)^{r}\right)^{[N / r]}$.)

Given any set of $r$ paths with $\eta\left(\omega^{N, 1}\right), \ldots, \eta\left(\omega^{N, r}\right)$ linearly independent, there is an $N$ independent number of possibilities to choose $\omega^{N, r+1}, \ldots, \omega^{N, l}$ so that $\eta\left(\omega^{N, r+1}\right), \ldots \eta\left(\omega^{N, l}\right)$ 
are linear combinations of $\eta\left(\omega^{N, 1}\right), \ldots, \eta\left(\omega^{N, r}\right)$. To see this, first consider the equation $\lambda_{1} \eta\left(\omega^{N, 1}\right)+\cdots+\lambda_{r} \eta\left(\omega^{N, r}\right)=0$ with unknown $\lambda_{1}, \ldots, \lambda_{r}$. For any moment of time $m \in[0, N]$ this means $\lambda_{1} \eta\left(m, \omega_{m}^{1}\right)+\cdots+\lambda_{r} \eta\left(m, \omega_{m}^{r}\right)=0$. If $\omega_{m}^{i_{1}}=\omega_{m}^{i_{2}}=\cdots \omega_{m}^{i_{k}}$ but $\omega_{m}^{j} \neq \omega_{m}^{i_{1}}$ for all $j \in\{1, \ldots, r\} \backslash\left\{i_{1}, \ldots, i_{k}\right\}$, then $\lambda_{i_{1}}+\cdots+\lambda_{i_{k}}=0$. Then for any $m \in[0, N]$ the equation $\lambda_{1} \eta\left(m, \omega_{m}^{1}\right)+\cdots+\lambda_{r} \eta\left(m, \omega_{m}^{r}\right)=0$ splits into a certain number $n(m)(1 \leq n(m) \leq r)$ equations of type $\lambda_{i_{1}}+\cdots+\lambda_{i_{k}}=0$. Let us construct a matrix $A$ with $r$ columns and at least $N$ and at most $r N$ rows in the following way. For any $m>0$, according to given $\omega_{m}^{1}, \ldots, \omega_{m}^{r}$, let us add to $\mathrm{A} n(m)$ rows : each equation $\lambda_{i_{1}}+\cdots+\lambda_{i_{k}}=0$ gives a row with 1 at places $i_{1}, \ldots, i_{k}$ and 0 at all other places. Then the equation $\lambda_{1} \eta\left(\omega^{N, 1}\right)+\cdots+\lambda_{r} \eta\left(\omega^{N, i}\right)=0$ is equivalent $A \vec{\lambda}=\overrightarrow{0}$ with $\vec{\lambda}=\left(\lambda_{1}, \ldots, \lambda_{r}\right)$. Since this equation has only a trivial solution $\vec{\lambda}=0$, then the rank of $A$ equals $r$. The matrix $A$ contains at most $2^{r}$ different rows. There is less than $\left(2^{r}\right)^{r}$ possibilities to choose $r$ linearly independent of them. Let $A^{r \times r}$ be an $r \times r$ matrix consisting of $r$ linearly independent rows of $A$. The fact that $\eta\left(\omega^{N, r+1}\right)$ is a linear combination $\mu_{1} \eta\left(\omega^{N, 1}\right)+\cdots+\mu_{r} \eta\left(\omega^{N, r}\right)=\eta\left(\omega^{N, r+1}\right)$ can be written as $A^{r \times r} \vec{\mu}=\vec{b}$ where the vector $\vec{b}$ contains only 1 and 0 : if a given row $t$ of the matrix $A^{r \times r}$ corresponds to the $m$ th step of the random walks and has 1 at places $i_{1}, \ldots, i_{k}$ and 0 elsewhere, then we put $b_{t}=1$ if $\omega_{m}^{i_{1}}=\omega_{m}^{r+1}$ and $b_{t}=0$ if $\omega_{m}^{i_{1}} \neq \omega_{m}^{r+1}$. Thus, given $\omega^{N, 1}, \ldots, \omega^{N, r}$, there is an $N$ independent number of possibilities to write the system $A^{r \times r} \vec{\mu}=\vec{b}$ with non degenerate matrix $A^{r \times r}$ which determines uniquely linear coefficients $\mu_{1}, \ldots, \mu_{r}$ and consequently $\eta\left(\omega^{N, r+1}\right)$ and the path $\omega^{N, r+1}$ itself through these linear coefficients. Hence, there is not more possibilities to choose $\omega^{N, r+1}$ than the number of non-degenerate matrices $A^{r \times r}$ multiplied by the number of vectors $\vec{b}$, that is roughly not more than $2^{r^{2}+r}$.

These observations lead to the fact that the sum (11) with the covariance matrix $B_{N}\left(\omega^{N, 1}, \ldots, \omega^{N, l}\right)$ of the rank $r$ contains at most $\left(2^{r^{2}+r}\right)^{l-r} 2^{N r} e^{-p N}$ different terms with some constant $p>0$. Then, taking into account the estimate (24) of each term with $2 \alpha<1$, we deduce that it converges to zero exponentially fast. This finishes the proof of (6).

To show (2), we have already noticed that the sum of terms $\mathrm{P}\left(\forall_{i=1}^{2}:\left|\eta\left(\omega^{N, i}\right)-E_{N}\right|<b_{i} \delta_{N}\right)$ over all pairs of different paths $\omega^{N, 1}, \omega^{N, 2}$ in $S_{N}^{l} \backslash \mathcal{R}_{N, l}^{\theta_{0}}$ converges to zero exponentially fast. Then (2) follows from the Borel-Cantelli lemma.

Proof of Theorem 3. We have again to verify the hypothesis of Theorem 4 for $V_{i, M}$ given by $\delta_{N}^{-1}\left|\eta\left(\omega^{N, i}\right)-E_{N}\right|$, i.e. we must show (11).

For $\beta \in] 0,1[$ let us denote by

$$
\mathcal{K}_{N, l}^{\beta}=\left\{\left(\omega^{N, 1}, \ldots, \omega^{N, l}\right): \operatorname{cov}\left(\eta\left(\omega^{N, i}\right), \eta\left(\omega^{N, j}\right)\right) \leq N^{\beta-1}, \forall i, j=1, \ldots, l, i \neq j\right\} .
$$

Step 1. In this step we estimate the cardinality of the complementary set to $\mathcal{K}_{N, l}^{\beta}$ in (26) and (27).

We have :

$$
\begin{aligned}
& \left|S_{N}^{l} \backslash \mathcal{K}_{N, l}^{\beta}\right| \\
& \quad \leq(l(l-1) / 2)(2 d)^{N(l-2)} \#\left\{\omega^{N, 1}, \omega^{N, 2}: \#\left\{m \in[0, \ldots, N]: \omega_{m}^{1}-\omega_{m}^{2}=0\right\}>N^{\beta}\right\} .
\end{aligned}
$$

It has been shown in the proof of Theorem 2 that the number

$$
\#\left\{\omega^{N, 1}, \omega^{N, 2}: \#\left\{m \in[0, \ldots, N]: \omega_{m}^{1}-\omega_{m}^{2}=0\right\}>N^{\beta}\right\}
$$


equals the number of paths of a simple random walk within the period $[0,2 N]$ that visit the origin at least $\left[N^{\beta}\right]+1$ times.

Let $W_{r}$ be the time of the $r$ th return to the origin of a simple random walk $\left(W_{1}=0\right), R_{N}$ be the number of returns to the origin in the first $N$ steps. Then for any integer $q$

$$
P\left(R_{N} \leq q\right)=P\left(W_{1}+\left(W_{2}-W_{1}\right)+\cdots+\left(W_{q}-W_{q-1}\right) \geq N\right) \geq \sum_{k=1}^{q-1} P\left(E_{k}\right)
$$

where $E_{k}$ is the event that exactly $k$ of the variables $W_{s}-W_{s-1}$ are greater than or equal to $N$, and $q-1-k$ are less than $N$. Then

$$
\begin{gathered}
\sum_{k=1}^{q-1} P\left(E_{k}\right)=\sum_{k=1}^{q-1}\left(\begin{array}{c}
q-1 \\
k
\end{array}\right) P\left(W_{2}-W_{1} \geq N\right)^{k}\left(1-P\left(W_{2}-W_{1} \geq N\right)\right)^{q-1-k} \\
=1-\left(1-P\left(W_{2}-W_{1} \geq N\right)\right)^{q-1}
\end{gathered}
$$

It is shown in [15] that in the case $d=2$

$$
P\left(W_{2}-W_{1} \geq N\right)=\pi(\log N)^{-1}\left(1+O\left((\log N)^{-1}\right)\right), \quad N \rightarrow \infty .
$$

Then

$$
P\left(R_{N}>q\right) \leq\left(1-\pi(\log N)^{-1}(1+o(1))\right)^{q-1}
$$

Consequently,

$$
\begin{gathered}
\#\left\{\omega^{N, 1}, \omega^{N, 2}: \#\left\{m \in[0, \ldots, N]: \omega_{m}^{1}-\omega_{m}^{2}=0\right\}>N^{\beta}\right\} \\
=(2 d)^{2 N} P\left(R_{2 N}>\left[N^{\beta}\right]\right) \\
\leq(2 d)^{2 N}\left(1-\pi(\log 2 N)^{-1}(1+o(1))\right)^{\left[N^{\beta}\right]-1} \leq(2 d)^{2 N} \exp \left(-h(\log 2 N)^{-1} N^{\beta}\right)
\end{gathered}
$$

with some constant $h>0$. Finally for $d=2$ and all $N>0$ by (25)

$$
\left|S_{N}^{l} \backslash \mathcal{K}_{N, l}^{\beta}\right| \leq(2 d)^{l N} \exp \left(-h_{2}(\log 2 N)^{-1} N^{\beta}\right)
$$

with some constant $h_{2}>0$.

In the case $d \geq 3$ the random walk is transient and

$$
P\left(W_{2}-W_{1} \geq N\right) \geq P\left(W_{2}-W_{1}=\infty\right)=\gamma_{d}>0 .
$$

It follows that $\mathrm{P}\left(R_{N}>q\right) \leq\left(1-\gamma_{d}\right)^{q-1}$ and consequently

$$
\left|S_{N}^{l} \backslash \mathcal{K}_{N, l}^{\beta}\right| \leq(2 d)^{l N} \exp \left(-h_{d} N^{\beta}\right)
$$

with some constant $h_{d}>0$.

Step 2. Proceeding exactly as in the proof of Theorem 2, we obtain that uniformly for $\left(\omega^{N, 1}, \ldots, \omega^{N, l}\right) \in \mathcal{K}_{N, l}^{\beta}$,

$$
\mathrm{P}\left(\forall_{i=1}^{l}:\left|\eta\left(\omega^{N, i}\right)-E_{N}\right|<b_{i} \delta_{N}\right)
$$




$$
=\left(2 \delta_{N} / \sqrt{2 \pi}\right)^{l}\left(b_{1} \cdots b_{l}\right) e^{-\left\|v e c E_{N}\right\|^{2}\left(1+O\left(N^{\beta-1}\right)\right) / 2}(1+o(1))
$$

where we denoted by $\vec{E}_{N}$ the vector $\left(E_{N}, \ldots, E_{N}\right)$. Moreover, if the covariance the matrix $B_{N}\left(\omega^{N, 1}, \ldots, \omega^{N, l}\right)$ is of the rank $r \leq l$ (using the fact that its determinant is a finite polynomial in the variables $1 / N)$ we get as in the proof of Theorem 2 that

$$
\mathrm{P}\left(\forall_{i=1}^{l}:\left|\eta\left(\omega^{N, i}\right)-E_{N}\right|<b_{i} \delta_{N}\right) \leq(2 d)^{-N r} e^{c^{2} r N^{2 \alpha} / 2} N^{k(r)}
$$

for some $k(r)>0$.

Step 3. Having (26), (27), (3) and (29), we are able to carry out the proof of the theorem. For given $\alpha \in] 0,1 / 2\left[\right.$, let us choose first $\beta_{0}>0$ such that

$$
2 \alpha-1+\beta_{0}<0 .
$$

Next, let us choose $\beta_{1}>\beta_{0}$ such that

$$
2 \alpha-1+\beta_{1}<\beta_{0}
$$

then $\beta_{2}>\beta_{1}$ such that

$$
2 \alpha-1+\beta_{2}<\beta_{1},
$$

etc. After $i-1$ steps we choose $\beta_{i}>\beta_{i-1}$ such that

$$
2 \alpha-1+\beta_{i}<\beta_{i-1} .
$$

Let us take e.g. $\beta_{i}=(i+1) \beta_{0}$. We stop the procedure at $n=\left[2 \alpha / \beta_{0}\right]$ th step, that is

$$
n=\min \left\{i \geq 0: 2 \alpha<\beta_{i}\right\}
$$

Note that $\beta_{n-1} \leq 2 \alpha$, and then $\beta_{n}=\beta_{n-1}+\beta_{0}<2 \alpha+1-2 \alpha=1$.

We will prove that the sum (11) over $\mathcal{K}_{N, l}^{\beta_{0}}$ converges to $b_{1} \cdots b_{l}$, while those over $\mathcal{K}_{N, l}^{\beta_{i}} \backslash \mathcal{K}_{N, l}^{\beta_{i-1}}$ for $i=1,2, \ldots, n$ and the one over $S_{N}^{l} \backslash \mathcal{K}_{N, l}^{\beta_{n}}$ converge to zero.

By (3), each term of the sum (11) over $\mathcal{K}_{N, l}^{\beta_{0}}$ equals

$$
\left(2 \delta_{N} / \sqrt{2 \pi}\right)^{l}\left(b_{1} \cdots b_{l}\right) e^{-\left\|\vec{E}_{N}\right\|^{2}\left(1+O\left(N^{\beta_{0}-1}\right)\right) / 2}(1+o(1)) .
$$

Here $e^{\left\|\vec{E}_{N}\right\|^{2} \times O\left(N^{\beta_{0}-1}\right)}=1+o(1)$ by the choice (30) of $\beta_{0}$. Then, by the definition of $\delta_{N}$ (8), each term of the sum (11) over $\mathcal{K}_{N, l}^{\beta_{0}}$ is

$$
\left(b_{1} \cdots b_{l}\right)(2 d)^{-N l}(1+o(1))
$$

uniformly for $\left(\omega^{N, 1}, \ldots, \omega^{N, l}\right) \in \mathcal{K}_{N, l}^{\eta_{0}}$. The number of terms in this sum is $\left|\mathcal{K}_{N, l}^{\beta_{0}}\right|$, that is $(2 d)^{N l}(1+o(1))$ by (26) and (27). Hence, the sum (11) over $\mathcal{K}_{N, l}^{\beta_{0}}$ converges to $b_{1} \cdots b_{l}$.

Let us consider the sum over $\mathcal{K}_{N, l}^{\beta_{i}} \backslash \mathcal{K}_{N, l}^{\beta_{i-1}}$ for $i=1,2, \ldots, n$. By (3) each term in this sum equals

$$
\left(2 \delta_{N} / \sqrt{2 \pi}\right)^{l}\left(b_{1} \cdots b_{l}\right) e^{-\left\|\vec{E}_{N}\right\|^{2}\left(1+O\left(N^{\beta_{i}-1}\right) / 2\right.}(1+o(1))
$$


uniformly for $\left(\omega^{N, 1}, \ldots, \omega^{N, l}\right) \in \mathcal{K}_{N, l}^{\beta_{i}}$. Then, by the definition of $\delta_{N}(8)$, it is bounded by the quantity $(2 d)^{-N l} C_{i} e^{h_{i} N^{2 \alpha-1+\beta_{i}}}$ with some constants $C_{i}, h_{i}>0$. The number of terms in this sum is not greater than $\left|S_{N}^{l} \backslash \mathcal{K}_{N, l}^{\beta_{i-1}}\right|$ which is bounded by $(2 d)^{N l} \exp \left(-h_{2} N^{\beta_{i-1}}(\log 2 N)^{-1}\right)$ in the case $d=2$ due to (26) and by the quantity $(2 d)^{N l} \exp \left(-h_{d} N^{\beta_{i-1}}\right)$ in the case $d \geq 3$ due to (27). Then by the choice of $\beta_{i}$ (33) this sum converges to zero exponentially fast.

Let us now treat the sum over $S_{N}^{l} \backslash \mathcal{K}_{N, l}^{\beta_{n}}$. Let us first analyze the sum over $\left(\omega^{N, 1}, \ldots, \omega^{N, l}\right)$ such that the matrix $B_{N}\left(\omega^{N, 1}, \ldots, \omega^{N, l}\right)$ is non-degenerate. By (29) each term in this sum is bounded by $(2 d)^{-N l} e^{c^{2} l N^{2 \alpha} / 2} N^{k(l)}$ for some $k(l)>0$. The number of terms in this sum is bounded by the quantity $(2 d)^{N l} \exp \left(-h_{2} N^{\beta_{n}}(\log 2 N)^{-1}\right)$ in the case $d=2$ and by $(2 d)^{N l} \exp \left(-h_{d} N^{\beta_{n}}\right)$ in the case $d \geq 3$ respectively by (26) and (27). Since $2 \alpha<\beta_{n}$ by (34), this sum converges to zero exponentially fast.

Let us finally turn to the sum over $\left(\omega^{N, 1}, \ldots, \omega^{N, l}\right)$ such that the matrix $B_{N}\left(\omega^{N, 1}, \ldots, \omega^{N, l}\right)$ is degenerate of the rank $r<l$. By (29) each term in this sum is bounded by $(2 d)^{-N r} e^{c^{2} r N^{2 \alpha} / 2} N^{k(r)}$ for some $k(r)>0$, while exactly by the same arguments as in the proof of Theorem 2, (they are, indeed, valid in all dimensions) the number of terms in this sum is less than $O\left((2 d)^{N r}\right) e^{-p N}$ with some constant $p>0$. Hence, this last sum converges to zero exponentially fast as $2 \alpha<1$. This finishes the proof of (9). The proof of (3) is completely analogous to the one of (2).

Proof of Theorem 1. We again concentrate on the proof in the sum (11) with $E_{N}=c$.

Step 1. First of all, we need a rather rough estimate of the probabilities of (11). Let $\left(\omega^{N, 1}, \ldots, \omega^{N, r}\right)$ be such that the matrix $B_{N}\left(\omega^{N, 1}, \ldots, \omega^{N, r}\right)$ is non-degenerate. We prove in this step that there exists a constant $k(r)>0$ such that for any $N>0$ and any $\left(\omega^{N, 1}, \ldots, \omega^{N, r}\right)$ with non-degenerate $B_{N}\left(\omega^{N, 1}, \ldots, \omega^{N, r}\right)$, we have:

$$
\mathrm{P}\left(\forall_{i=1}^{r}:\left|\eta\left(\omega^{N, i}\right)-c\right|<b_{i} \delta_{N}\right) \leq(2 d)^{-N r} N^{k(r)} .
$$

Let

$$
f_{N}^{\omega^{N, 1}, \ldots, \omega^{N, r}}\left(t_{1}, \ldots, t_{r}\right)=\mathrm{E} \exp \left(i \sum_{k=1}^{r} t_{k} \eta\left(\omega^{N, k}\right)\right)
$$

be the Fourier transform of $\left(\eta\left(\omega^{N, 1}\right), \ldots, \eta\left(\omega^{N, r}\right)\right)$. Then

$$
\begin{aligned}
\mathrm{P} & \left(\forall_{i=1}^{r}:\left|\eta\left(\omega^{N, i}\right)-c\right|<b_{i} \delta_{N}\right) \\
& =\frac{1}{(2 \pi)^{r}} \int_{\mathbf{R}^{r}} f_{N}^{\omega^{N, 1}, \ldots, \omega^{N, r}}(\vec{t}) \prod_{k=1}^{r} \frac{e^{-i t_{k}\left(-b_{k} \delta_{N}+c\right)}-e^{-i t_{k}\left(b_{k} \delta_{N}+c\right)}}{i t_{k}} d t_{1} \cdots d t_{r}
\end{aligned}
$$

provided that the integrand is in $L^{1}\left(\mathbf{R}^{d}\right)$. We will show that this is the case due to the assumption made on $\phi$ and deduce the bound (35).

We know that the function $f_{N}^{\omega^{N, 1}, \ldots, \omega^{N, r}}(\vec{t})$ is the product of $N$ Fourier transforms :

$$
f_{N}^{\omega^{N, 1}, \ldots, \omega^{N, r}}(\vec{t})=\prod_{n=1}^{N} \mathrm{E} \exp \left(i N^{-1 / 2} \sum_{k=1}^{r} t_{k} \eta\left(n, \omega_{n}^{N, k}\right)\right) .
$$

Moreover, each of these functions is itself a product of (at minimum 1 and at maximum $r$ ) Fourier transforms of type $\phi\left(\left(t_{i_{1}}+\cdots+t_{i_{k}}\right) N^{-1 / 2}\right)$. More precisely, let us construct the matrix $A$ with $r$ 
columns and at least $N$ and at most $r N$ rows as in the proof of Theorem 2. Namely, for each step $n=0,1,2, \ldots, N$, we add to the matrix $A$ at least 1 and at most $r$ rows according to the following rule: if $\omega_{n}^{N, i_{1}}=\omega_{n}^{N, i_{2}}=\cdots=\omega_{n}^{N, i_{k}}$ and $\omega_{n}^{N, j} \neq \omega_{n}^{N, i_{1}}$ for any $j \in\{1, \ldots, r\} \backslash\left\{i_{1}, \ldots, i_{k}\right\}$, we add to $A$ a row with 1 at places $i_{1}, \ldots, i_{k}$ and 0 at other $r-k$ places. Then

$$
f_{N}^{\omega^{N, 1}, \ldots, \omega^{N, r}}(\vec{t})=\prod_{j} \phi\left(N^{-1 / 2}(A \vec{t})_{j}\right) .
$$

Since $B_{N}\left(\omega^{N, 1}, \ldots, \omega^{N, r}\right)$ is non-degenerate, the rank of the matrix $A$ equals $r$. Let us choose in $A$ any $r$ linearly independent rows, and let us denote by $A^{r}$ the $r \times r$ matrix constructed by them. Then by the assumption made on $\phi$

$$
\left|f_{N}^{\omega^{N, 1}, \ldots, \omega^{N, r}}(\vec{t})\right| \leq \prod_{j=1}^{r}\left|\phi\left(N^{-1 / 2}\left(A^{r} \vec{t}\right)_{j}\right)\right| \leq \prod_{j=1}^{r} \min \left(1, \frac{C N^{1 / 2}}{\left|\left(A^{r} \vec{t}\right)_{j}\right|}\right) \leq C^{r} N^{r / 2} \prod_{j=1}^{r} \min \left(1, \frac{1}{\left|\left(A^{r} \vec{t}\right)_{j}\right|}\right)
$$

with some constant $C>0$. Furthermore

$$
\left|\prod_{k=1}^{r} \frac{e^{-i t_{k}\left(-b_{k} \delta_{N}+c\right)}-e^{-i t_{k}\left(b_{k} \delta_{N}+c\right)}}{i t_{k}}\right| \leq \prod_{k=1}^{r} \min \left(\left(2 \delta_{N}\right) b_{k}, \frac{2}{\left|t_{k}\right|}\right) \leq C^{\prime} \prod_{k=1}^{r} \min \left((2 d)^{-N}, \frac{1}{\left|t_{k}\right|}\right)
$$

with some $C^{\prime}>0$. Hence,

$$
\begin{aligned}
& \frac{1}{(2 \pi)^{r}} \int_{\mathbf{R}^{r}}\left|f_{N}^{\omega^{N, 1}, \ldots, \omega^{N, r}}(\vec{t}) \prod_{k=1}^{r} \frac{e^{-i t_{k}\left(-b_{k} \delta_{N}+c\right)}-e^{-i t_{k}\left(b_{k} \delta_{N}+c\right)}}{i t_{k}}\right| d t_{1} \cdots d t_{r} \\
& \leq C_{0} N^{r / 2} \int \prod_{k=1}^{r} \min \left((2 d)^{-N}, \frac{1}{\left|t_{k}\right|}\right) \min \left(1, \frac{1}{\left|\left(A^{r} \vec{t}\right)_{k}\right|}\right) d \vec{t}
\end{aligned}
$$

with some constant $C_{0}>0$ depending on the function $\phi$ and on $b_{1}, \ldots, b_{r}$ only. Since the matrix $A^{r}$ is non-degenerate, using easy arguments of linear algebra, one can show that for some constant $C_{1}>0$ depending on the matrix $A^{r}$ only, we have

$$
\int \prod_{k=1}^{r} \min \left((2 d)^{-N}, \frac{1}{\left|t_{k}\right|}\right) \min \left(1, \frac{1}{\left|\left(A^{r} \vec{t}\right)_{k}\right|}\right) d \vec{t} \leq C_{1} \int \prod_{k=1}^{r} \min \left((2 d)^{-N}, \frac{1}{\left|t_{k}\right|}\right) \min \left(1, \frac{1}{\left|t_{k}\right|}\right) d \vec{t} .
$$

The proof of (42) is given in Appendix. But the right-hand of (42) is finite. This shows that the integrand in (36) is in $L^{1}\left(\mathbf{R}^{d}\right)$ and the inversion formula (36) is valid. Moreover, the right-hand side of (42) equals $C_{1}\left(2\left((2 d)^{-N}+(2 d)^{-N} N \ln 2 d+(2 d)^{-N}\right)\right)^{r}$. Hence, the probabilities above are bounded by the quantity $C_{0} N^{r / 2} C_{1} 2^{r}(2+N \ln (2 d))^{r}(2 d)^{-N r}$ with $C_{0}$ depending on $\phi$ and $b_{1}, \ldots, b_{r}$ and $C_{1}$ depending on the choice of $A^{r}$. To conclude the proof of (35), it remains to remark that there is an $N$-independent number of possibilities to construct a matrix $A^{r}$ (at most $2^{r^{2}}$ ), since it contains only 0 or 1 .

Step 2. We keep the notation $\mathcal{R}_{N, l}^{\theta}$ from (3) for $\left.\theta \in\right] 0,1 / 2[$. The cardinality of the complementary set for $d=1$ is estimated in (14). Moreover by (26) for $d=2$

$$
\left|S_{N}^{l} \backslash \mathcal{R}_{N, l}^{\theta}\right|=\left|S_{N}^{l} \backslash \mathcal{K}_{N, l}^{\theta+1 / 2}\right| \leq(2 d)^{N l} \exp \left(-h_{2}(\log 2 N)^{-1} N^{1 / 2+\theta}\right)
$$


and by (27) for $d \geq 3$

$$
\left|S_{N}^{l} \backslash \mathcal{R}_{N, l}^{\theta}\right|=\left|S_{N}^{l} \backslash \mathcal{K}_{N, l}^{\theta+1 / 2}\right| \leq(2 d)^{N l} \exp \left(-h_{d} N^{1 / 2+\theta}\right)
$$

so that, for all $d \geq 1$ there are $h_{d}, C_{d}>0$ such that for all $N>0$

$$
\left|S_{N}^{l} \backslash \mathcal{R}_{N, l}^{\theta}\right| \leq(2 d)^{N l} C_{d} N \exp \left(-h_{d} N^{2 \theta}\right) .
$$

Sep 3. In this step we show that uniformly for $\left(\omega^{N, 1}, \ldots, \omega^{N, l}\right) \in \mathcal{R}_{N, l}^{\theta}$

$$
\mathrm{P}\left(\forall_{i=1}^{l}:\left|\eta\left(\omega^{N, i}\right)-c\right|<b_{i} \delta_{N}\right)=(2 d)^{-N l} b_{1} \cdots b_{l}(1+o(1)) .
$$

For any $\left(\omega^{N, 1}, \ldots \omega^{N, l}\right) \in \mathcal{R}_{N, l}^{\theta}$, we can represent the probabilities in the sum (11) as sums of four terms :

$$
\begin{aligned}
\mathrm{P} & \left.\forall_{i=1}^{l}:\left|\eta\left(\omega^{N, i}\right)-c\right|<b_{i} \delta_{N}\right) \\
& =\frac{1}{(2 \pi)^{l}} \int_{\mathbf{R}^{l}} f_{N}^{\omega^{N, 1}, \ldots, \omega^{N, l}}(\vec{t}) \prod_{k=1}^{l} \frac{e^{-i t_{k}\left(-b_{k} \delta_{N}+c\right)}-e^{-i t_{k}\left(b_{k} \delta_{N}+c\right)}}{i t_{k}} d t_{1} \cdots d t_{l} \\
& =\sum_{m=1}^{4} I_{N}^{m}\left(\omega^{N, 1}, \ldots, \omega^{N, l}\right)
\end{aligned}
$$

where

$$
\begin{aligned}
I_{N}^{1}= & \frac{1}{(2 \pi)^{l}} \int_{\mathbf{R}^{l}} \prod_{k=1}^{l} \frac{e^{-i t_{k}\left(-b_{k} \delta_{N}+c\right)}-e^{-i t_{k}\left(b_{k} \delta_{N}+c\right)}}{i t_{k}} e^{-\vec{t} B_{N}\left(\omega^{N, 1}, \ldots, \omega^{N, l}\right) \vec{t} / 2} d \vec{t} \\
& -\frac{1}{(2 \pi)^{l}} \int_{\|t\|>\epsilon N^{1 / 6}} \prod_{k=1}^{l} \frac{e^{-i t_{k}\left(-b_{k} \delta_{N}+c\right)}-e^{-i t_{k}\left(b_{k} \delta_{N}+c\right)}}{i t_{k}} e^{-\vec{t} B_{N}\left(\omega^{N, 1}, \ldots, \omega^{N, l}\right) \vec{t} / 2} d \vec{t} . \\
I_{N}^{2}= & \frac{1}{(2 \pi)^{l}} \int_{\|t\|<\epsilon N^{1 / 6}} \prod_{k=1}^{l} \frac{e^{-i t_{k}\left(-b_{k} \delta_{N}+c\right)}-e^{-i t_{k}\left(b_{k} \delta_{N}+c\right)}}{i t_{k}} \times\left(f_{N}^{\omega^{N, 1}, \ldots, \omega^{N, l}}(\vec{t})-e^{-\vec{t} B_{N}\left(\omega^{N, 1}, \ldots, \omega^{N, l}\right) \vec{t} / 2}\right) d \vec{t} \\
I_{N}^{3}= & \frac{1}{(2 \pi)^{l}} \int_{\epsilon N^{1 / 6}<\|t\|<\delta N^{1 / 2}} \int_{k=1}^{l} \frac{e^{-i t_{k}\left(-b_{k} \delta_{N}+c\right)}-e^{-i t_{k}\left(b_{k} \delta_{N}+c\right)}}{i t_{N}} f^{\omega^{N, 1}, \ldots, \omega^{N, l}}(\vec{t}) d \vec{t} \\
I_{N}^{4}= & \frac{1}{(2 \pi)^{l}} \int_{\|t\|>\delta N^{1 / 2}} \prod_{k=1}^{l} \frac{e^{-i t_{k}\left(-b_{k} \delta_{N}+c\right)}-e^{-i t_{k}\left(b_{k} \delta_{N}+c\right)}}{i t_{N}} f_{N, 1, \ldots, \omega^{N, l}}(\vec{t}) d \vec{t}
\end{aligned}
$$

with $\epsilon, \delta>0$ chosen according to the following Proposition 1 .

Proposition 1. There exist constants $N_{0}, C, \epsilon, \delta, \zeta>0$ such that for all $\left(\omega^{N, 1}, \ldots \omega^{N, l}\right) \in \mathcal{R}_{N, l}^{\theta}$ and all $N \geq N_{0}$ the following estimates hold:

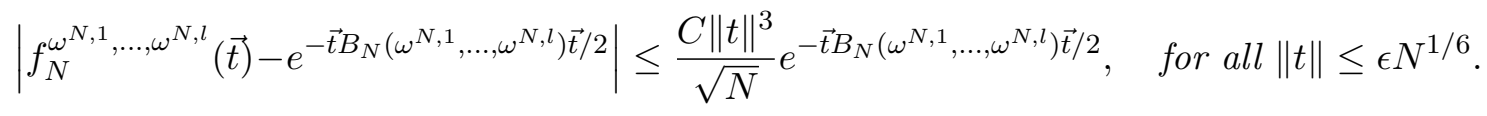




$$
\left|f_{N}^{\omega^{N, 1}, \ldots, \omega^{N, l}}(\vec{t})\right| \leq e^{-\zeta\|t\|^{2}} \quad \text { for all }\|t\|<\delta \sqrt{N}
$$

The proof of this proposition mimics the one of the Berry-Essen inequality and is given in Appendix.

The first part of $I_{N}^{1}$ is just the probability that $l$ Gaussian random variables with zero mean and covariance matrix $B_{N}\left(\omega^{N, 1}, \ldots, \omega^{N, l}\right)$ belong to the intervals $\left[-\delta_{N} b_{k}+c, \delta_{N} b_{k}+c\right]$ for $k=1, \ldots, l$ respectively. This is

$$
\begin{gathered}
\int_{\left|z_{j}-c\right| \leq \delta_{N} b_{j}, \forall_{j=1}^{l}} \frac{e^{-\left(\vec{z} B^{-1}\left(\omega^{N, 1}, \ldots, \omega^{N, l}\right) \vec{z}\right) / 2}}{(2 \pi)^{l / 2} \sqrt{\operatorname{det} B\left(\omega^{N, 1}, \ldots, \omega^{N, l}\right)}} d \vec{z} \\
=\left(2 \delta_{N} / \sqrt{2 \pi}\right)^{l}\left(b_{1} \cdots b_{l}\right) e^{-\left(\vec{c} B^{-1}\left(\omega^{N, 1}, \ldots, \omega^{N, l}\right) \vec{c}\right) / 2}(1+o(1)) \\
=\left(2 \delta_{N} / \sqrt{2 \pi}\right)^{l}\left(b_{1} \cdots b_{l}\right) e^{-l c^{2}\left(1+O\left(N^{\theta-1 / 2}\right)\right) / 2}(1+o(1))=(2 d)^{-N l} b_{1} \cdots b_{l}(1+o(1))
\end{gathered}
$$

uniformly for $\left(\omega^{N, 1}, \ldots, \omega^{N, l}\right) \in \mathcal{R}_{N, l}^{\theta}$, where we denoted by $\vec{c}$ the vector $(c, \ldots, c)$. Since

$$
\prod_{k=1}^{l}\left|\frac{e^{-i t_{k}\left(-b_{k} \delta_{N}+c\right)}-e^{-i t_{k}\left(b_{k} \delta_{N}+c\right)}}{i t_{k}}\right| \leq\left(2 \delta_{N} b_{1}\right) \cdots\left(2 \delta_{N} b_{l}\right)=O\left((2 d)^{-N l}\right)
$$

and the elements of the matrix $B_{N}\left(\omega^{N, 1}, \ldots, \omega^{N, l}\right)$ out of the diagonal are $O\left(N^{\theta-1 / 2}\right)=o(1)$ as $N \rightarrow \infty$, the second part of $I_{N}^{1}$ is smaller than $(2 d)^{-N l}$ exponentially (with exponential term $\exp \left(-h N^{1 / 3}\right)$ for some $\left.h>0\right)$.

There is a constant $C>0$ such that the term $I_{N}^{2}$ is bounded by $C(2 d)^{-N l} N^{-1 / 2}$ for any $\left(\omega^{N, 1}, \ldots \omega^{N, l}\right) \in \mathcal{R}_{N, l}^{\theta}$ and all $N$ large enough. This follows from (51), the estimate (48) and again the fact that the elements of the matrix $B_{N}\left(\omega^{N, 1}, \ldots, \omega^{N, l}\right)$ out of the diagonal are $O\left(N^{\theta-1 / 2}\right)=o(1)$ as $N \rightarrow \infty$.

The third term $I_{N}^{3}$ is exponentially smaller than $(2 d)^{-N l}$ by (51) and the estimate (49).

Finally, by (51)

$$
\left|I_{N}^{4}\right| \leq\left(2 \delta_{N} b_{1}\right) \cdots\left(2 \delta_{N} b_{l}\right) \int_{\|t\|>\delta \sqrt{N}}\left|f_{N}^{\omega^{N, 1}, \ldots, \omega^{N, l}}(\vec{t})\right| d \vec{t}=O\left((2 d)^{-N l}\right) \int_{\|t\|>\delta \sqrt{N}}\left|f_{N}^{\omega^{N, 1}, \ldots, \omega^{N, l}}(\vec{t})\right| d \vec{t} .
$$

The function $f_{N}^{\omega^{N, 1}, \ldots, \omega^{N, l}}(\vec{t})$ is the product of $N$ Fourier transforms (37). Note that for any pair $\omega^{N, i}, \omega^{N, j}$ of $\left(\omega^{N, 1}, \ldots, \omega^{N, l}\right) \in \mathcal{R}_{N, l}^{\theta}$, there are at most $N^{\theta+1 / 2}$ steps $n$ where $\omega_{n}^{N, i}=\omega_{n}^{N, j}$. Then there are at least $N-[l(l-1) / 2] N^{\theta+1 / 2}=a(N)$ steps where all $l$ coordinates $\omega^{N, i}, i=1, \ldots, l$, of the vector $\left(\omega^{N, 1}, \ldots, \omega^{N, l}\right) \in \mathcal{R}_{N, l}^{\theta}$ are different. In this case

$$
\mathrm{E} \exp \left(i N^{-1 / 2} \sum_{k=1}^{l} t_{k} \theta\left(n, \omega_{n}^{N, k}\right)\right)=\phi\left(t_{1} N^{-1 / 2}\right) \cdots \phi\left(t_{k} N^{-1 / 2}\right)
$$


By the assumption made on $\phi$, this function is aperiodic and thus $|\phi(t)|<1$ for $t \neq 0$. Moreover, for any $\delta>0$ there exists $h(\delta)>0$ such that $|\phi(t)| \leq 1-h(\delta)$ for $|t|>\delta / l$. Then

$$
\begin{gathered}
\int_{\|t\|>\delta \sqrt{N}}\left|f_{N}^{\omega^{N, 1}, \ldots, \omega^{N, l}}(\vec{t})\right| d \vec{t} \leq \int_{\|t\|>\delta \sqrt{N}}\left|\phi\left(t_{1} N^{-1 / 2}\right) \cdots \phi\left(t_{k} N^{-1 / 2}\right)\right|^{a(N)} d \vec{t} \\
=N^{l / 2} \int_{\|s\|>\delta}\left|\phi\left(s_{1}\right) \cdots \phi\left(s_{k}\right)\right|^{a(N)} d \vec{s} \leq N^{l / 2}(1-h(\delta))^{a(N)-2} \int_{\|s\|>\delta}\left|\phi\left(s_{1}\right) \cdots \phi\left(s_{k}\right)\right|^{2} d \vec{s}
\end{gathered}
$$

where $a(N)=N(1+o(1))$ and the last integral converges due to the assumption made on $\phi(s)$. Hence $I_{N}^{4}$ is exponentially smaller than $(2 d)^{-N l}$. This finishes the proof of (44).

Step 4. We are now able to prove the theorem using the estimates (35),(43) and (44). By (44), the sum (11) over $\mathcal{R}_{N, l}^{\theta}$ (with fixed $\left.\theta \in\right] 0,1 / 2\left[\right.$ ) that contains by $(43)(2 d)^{N l}(1+o(1)$ ) terms, converges to $b_{1} \cdots b_{l}$. The sum (11) over $\left(\omega^{N, 1}, \ldots, \omega^{N, l}\right) \notin \mathcal{R}_{N, l}^{\theta}$ but with $B_{N}\left(\omega^{N, 1}, \ldots, \omega^{N, l}\right)$ non-degenerate, by (43) has only at most $(2 d)^{N l} C N \exp \left(-h N^{2 \theta}\right)$ terms, while each of its terms by (35) with $r=l$ is of the order $(2 d)^{-N l}$ up to a polynomial term. Hence, this sum converges to zero. Finally, due to the fact that in any set $\left(\omega^{N, 1}, \ldots, \omega^{N, l}\right)$ taken into account in (11) the paths are all different, the sum over $\left(\omega^{N, 1}, \ldots, \omega^{N, l}\right) \notin \mathcal{R}_{N, l}^{\theta}$ with $B_{N}\left(\omega^{N, 1}, \ldots, \omega^{N, l}\right)$ of the rank $r<l$ has an exponentially smaller number of terms than $(2 d)^{N r}$. This has been shown in detail in the proof of Theorem 2 where the arguments did not depend on the dimension of the random walk. Since by (35) each of these terms is of the order $(2 d)^{-N r}$ up to a polynomial term, this sum converges to zero. This concludes the proof of (3). The proof of (1) is completely analogous to the one of (2).

\section{Appendix}

Proof of (42). It is carried out via trivial arguments of linear algebra.

Let $m=1,2, \ldots, r+1, D_{m-1}$ be a non-degenerate $r \times r$ matrix with the first $m-1$ rows having 1 on the diagonal and 0 outside of the diagonal. (Clearly, $D_{0}$ is just a non-degenerate matrix and $D_{r}$ is the diagonal matrix with 1 everywhere on the diagonal.) Let us introduce the integral

$$
\begin{aligned}
& J^{m-1}\left(D_{m-1}\right) \\
& =\int \prod_{k=1}^{r} \min \left((2 d)^{-N}, \frac{1}{\left|t_{k}\right|}\right) \min \left(1, \frac{1}{\left|\left(D_{m-1} \vec{t}\right)_{k}\right|}\right) d \vec{t} \\
& =\int \prod_{k=1}^{m-1} \min \left((2 d)^{-N}, \frac{1}{\left|t_{k}\right|}\right) \min \left(1, \frac{1}{\left|t_{k}\right|}\right) \prod_{k=m}^{r} \min \left((2 d)^{-N}, \frac{1}{\left|t_{k}\right|}\right) \min \left(1, \frac{1}{\left|\left(D_{m-1} \vec{t}\right)_{k}\right|}\right) d \vec{t} .
\end{aligned}
$$

Sice $D_{m-1}$ is non-degenerate, there exists $i \in\{m, \ldots, r\}$ such that $d_{m, i} \neq 0$ and the matrix $D_{m}$ which is obtained from the matrix $D_{m-1}$ by replacing its $m$ th row by the one with 1 at the place $(m, i)$ and 0 at all places $(m, j)$ for $j \neq i$ is non-degenerate. Without loss of generality we may assume that $i=m$ (otherwise juste permute the $m$ th with the $i$ th column in $D_{m-1}$ and $t_{i}$ with $t_{m}$ in the integral $J^{m-1}\left(D_{m-1}\right)$ above). Since either $\left|t_{m-1}\right|<\left|\left(D_{m-1} \vec{t}\right)_{m-1}\right|$ or 
$\left|t_{m-1}\right| \geq\left|\left(D_{m-1} \vec{t}\right)_{m-1}\right|$, we can estimate $J^{m-1}\left(D_{m-1}\right)$ roughly by the sum of the following two terms :

$$
\begin{aligned}
& J^{m-1}\left(D_{m-1}\right) \\
& \leq \int \prod_{k=1}^{m} \min \left((2 d)^{-N}, \frac{1}{\left|t_{k}\right|}\right) \min \left(1, \frac{1}{\left|t_{k}\right|}\right) \prod_{k=m+1}^{r} \min \left((2 d)^{-N}, \frac{1}{\left|t_{k}\right|}\right) \min \left(1, \frac{1}{\left|\left(D_{m-1} \vec{t}\right)_{k}\right|}\right) d \vec{t} \\
& \quad+\int \prod_{k=1}^{m-1} \min \left((2 d)^{-N}, \frac{1}{\left|t_{k}\right|}\right) \min \left(1, \frac{1}{\left|t_{k}\right|}\right) \min \left((2 d)^{-N}, \frac{1}{\left|\left(D_{m-1} \vec{t}\right)_{m}\right|}\right) \min \left(1, \frac{1}{\left|\left(D_{m-1} \vec{t}\right)_{m}\right|}\right) \\
& \quad \times \prod_{k=m+1}^{r} \min \left((2 d)^{-N}, \frac{1}{\left|t_{k}\right|}\right) \min \left(1, \frac{1}{\left|\left(D_{m-1} \vec{t}\right)_{k}\right|}\right) d \vec{t} .
\end{aligned}
$$

The first term here is just $J^{m}\left(D_{m}\right)$. Let us make a change of variables in the second one : let $\vec{z}=B_{D_{m-1}} \vec{t}$, where the matrix $B_{D_{m-1}}$ is chosen such that $z_{1}=t_{1}, \ldots, z_{m-1}=t_{m-1}, z_{m}=$ $\left(D_{m-1} \vec{t}\right)_{m}, z_{m+1}=t_{m+1}, \ldots, z_{r}=t_{r}$. (Clearly, its $m$ th row is the same as in the matrix $D_{m-1}$, and it has 1 on the diagonal in all other $r-1$ rows and 0 outside of it.) Since $d_{m, m} \neq 0$, the matrix $B$ is non-degenerate. Then $D_{m-1} \vec{t}=D_{m-1} B_{D_{m-1}}^{-1} \vec{z}$, where the matrix $D_{m-1} B_{D_{m-1}}^{-1}$ is non-degenerate, and, moreover, it has the first $m$ rows with 1 on the diagonal and 0 outside of it, as we have $\left(D_{m-1} \vec{t}\right)_{1}=t_{1}=z_{1}, \ldots,\left(D_{m-1} \vec{t}\right)_{m-1}=t_{m-1}=z_{m-1},\left(D_{m-1} \vec{t}\right)_{m}=z_{m}$. Then (52) can be written as

$$
J^{m-1}\left(D_{m-1}\right) \leq J^{m}\left(D_{m}\right)+d_{m, m}^{-1} J^{m}\left(D_{m-1} B_{D_{m-1}}^{-1}\right) .
$$

Now, observe that the left-hand side of (42) is $J^{0}\left(A^{r}\right)$. By (53) it is bounded by $J^{1}\left(A_{1}^{r}\right)+$ $a_{1,1}^{-1} J^{1}\left(A^{r} B_{A^{r}}^{-1}\right)$. Again by (53) each of these two terms can be estimated by a sum of two terms of type $J^{2}(\cdot)$ etc. After $2^{r}$ applications of (53) $J^{0}\left(A^{r}\right)$ is bounded by a sum of $2^{r}$ terms of type $J^{r}\left(D_{r}\right)$ multiplied by some constants depending only on the initial matrix $A_{r}$. But all these $2^{r}$ terms $J^{r}\left(D_{r}\right)$ are the same as in the right-hand side of (42).

Proof of Proposition 1. We use the representation (38) of $f_{N}^{\omega^{N, 1}, \ldots, \omega^{N, l}}(\vec{t})$ as the product of a certain number $K\left(N, \omega^{N, 1}, \ldots, \omega^{N, l}\right)$ (denote it shortly by $K(N, \omega)$, clearly $N \leq K(N, \omega) \leq l N$ ) of Fourier transforms $\phi\left(N^{-1 / 2}(A \vec{t})_{j}\right)$ where at most $2^{l}$ are different. Each of them is of the form $\mathrm{E} \exp \left(i N^{-1 / 2}\left(t_{i_{1}}+\cdots+t_{i, k}\right) X\right)$ with $X$ a standard Gaussian random variable. Applying the fact that $\left|e^{i z}-1-i z-(i z)^{2} / 2 !\right| \leq|z|^{3} / 3$ ! for any $z \in \mathbf{R}$, we can write

$$
\phi\left(N^{-1 / 2}(A \vec{t})_{j}\right)=1-\frac{\left((A \vec{t})_{j}\right)^{2}}{2 ! N}-\nu_{j} \frac{\left((A \vec{t})_{j}\right)^{3} \mathrm{E}|X|^{3}}{3 ! N^{3 / 2}} \equiv 1-\alpha_{j}
$$

with some complex $\nu_{j}$ with $\left|\nu_{j}\right|<1$. It follows that there are some constants $C_{1}, C_{2}>0$ such that for any $\left(\omega^{N, 1}, \ldots, \omega^{N, l}\right) \in \mathcal{R}_{N, l}^{\theta}$ and any $j$ we have: $\left|\alpha_{j}\right| \leq C_{1}\|\vec{t}\|^{2} N^{-1}+C_{2}\|\vec{t}\|^{3} N^{-3 / 2}$. Then $\left|\alpha_{j}\right|<1 / 2$ and $\left|\alpha_{j}\right|^{2} \leq C_{3}\|\vec{t}\|^{3} N^{-3 / 2}$ with some $C_{3}>0$ for all $\vec{t}$ of the absolute value $\|\vec{t}\| \leq \delta \sqrt{N}$ with $\delta>0$ small enough. Thus $\ln \phi\left(N^{-1 / 2}(A \vec{t})_{j}\right)=-\alpha_{j}+\tilde{\nu}_{j} \alpha_{j}^{2} / 2$ (using the expansion $\ln (1+z)=z+\tilde{\nu} z^{2} / 2$ with some $\tilde{\nu}$ of the absolute value $|\tilde{\nu}|<1$ which is true for all $z$ with $|z|<1 / 2)$ for all $\left(\omega^{N, 1}, \ldots, \omega^{N, l}\right) \in \mathcal{R}_{N, l}^{\theta}$ and for all $\vec{t}$ with $\|\vec{t}\| \leq \delta \sqrt{N}$ with some $\tilde{\nu}_{j}$ such that $\left|\tilde{\nu}_{j}\right|<1$. It follows that

$$
f_{N}^{\omega^{N, 1}, \ldots, \omega^{N, l}}(\vec{t})=\exp \left(-\sum_{j=1}^{K(N, \omega)} \alpha_{j}+\sum_{j=1}^{K(N, \omega)} \tilde{\nu}_{j} \alpha_{j}^{2} / 2\right) .
$$


Since $A^{*} A=B_{N}\left(\omega^{N, 1}, \ldots, \omega^{N, l}\right)$, here $-\sum_{j=1}^{K(N, \omega)} \alpha_{j}=-\vec{t} B_{N}\left(\omega^{N, 1}, \ldots, \omega^{N, l}\right) \vec{t} / 2+\sum_{j=1}^{K(N, \omega)} p_{j}$ where $\left|p_{j}\right| \leq C_{2}\|\vec{t}\|^{3} N^{-3 / 2}$. Then

$$
f_{N}^{\omega^{N, 1}, \ldots, \omega^{N, l}}(\vec{t})=\exp \left(-\vec{t} B_{N}\left(\omega^{N, 1}, \ldots, \omega^{N, l}\right) \vec{t} / 2\right) \exp \left(\sum_{j=1}^{K(N, \omega)} p_{j}+\tilde{\nu}_{j} \alpha_{j}^{2} / 2\right)
$$

where $\left|p_{j}\right|+\left|\tilde{\nu}_{j} \alpha_{j}^{2} / 2\right| \leq\left(C_{2}+C_{3} / 2\right)\|\vec{t}\|^{3} N^{-3 / 2}$ for all $j$. Since $K(N, \omega) \leq l N$, we have

$$
\left|\sum_{j=1}^{K(N, \omega)} p_{j}+\tilde{\nu}_{j} \alpha_{j}^{2} / 2\right| \leq\left(C_{2}+C_{3} / 2\right) l\|t\|^{3} N^{-1 / 2}
$$

It follows that for $\epsilon>0$ small enough $\left|\exp \left(\sum_{j=1}^{K(N, \omega)} p_{j}+\tilde{\nu}_{j} \alpha_{j}^{2} / 2\right)-1\right| \leq C_{4}\|\vec{t}\|^{3} N^{-1 / 2}$ for all $\vec{t}$ with $\|\vec{t}\| \leq \epsilon N^{1 / 6}$. This proves (48). Finally

$$
\left|f_{N}^{\omega^{N, 1}, \ldots, \omega^{N, l}}(\vec{t})\right| \leq \exp \left(-\vec{t} B_{N}\left(\omega^{N, 1}, \ldots, \omega^{N, l}\right) \vec{t} / 2\right) \exp \left(\left(C_{2}+C_{3} / 2\right) l\|t\|^{3} N^{-1 / 2}\right) .
$$

Taking into account the fact that the elements of $B_{N}\left(\omega^{N, 1}, \ldots, \omega^{N, l}\right)$ out of the diagonal are at most $N^{-1 / 2+\theta}=o(1)$ as $N \rightarrow \infty$, one deduces from (58) that for $\delta>0$ small enough (49) holds true with some $\zeta>0$ for all $N$ large enough and all $\vec{t}$ with $\|\vec{t}\| \leq \delta \sqrt{N}$.

\section{References}

[1] H. Bauke, S. Franz, S. Mertens. Number partitioning as random energy model. Journal of Stat. Mech. : Theory and Experiment, page P04003 (2004).

[2] H. Bauke, S. Mertens. Universality in the level statistics of disordered systems. Phys. Rev. E 70, 025102(R) (2004).

[3] G. Ben Arous, V. Gayrard, A. Kuptsov. A new REM conjecture. Preprint (2006).

[4] E. Bolthausen. A note on diffusion of directed polymers in a random environment, Commun. Math. Phys. 123, 529-534, (1989). MR1006293

[5] C. Borgs, J. Chayes and B. Pittel. Phase transition and finite-size scaling for the integer partitioning problem. Random Structures and Algorithms 19, 247-288 (2001). MR1871556

[6] C. Borgs, J. Chayes, S. Mertens and C. Nair. Proof of the local REM conjecture for number partitioning I: Constant energy scales. Preprint (2005). To appear in Random Structures and Algorithms

[7] C. Borgs, J. Chayes, S. Mertens and C. Nair. Proof of the local REM conjecture for number partitioning II: Growing energy scales. Preprint (2005). To appear in Random Structures and Algorithms

[8] C. Borgs, J. Chayes, S. Mertens and B. Pittel. Phase diagram for the constrained integer partitioning problem. Random Structures and Algorithms 24, 315-380 (2004). MR2068872 
[9] A. Bovier, I. Kurkova. Poisson convergence in the restricted $k$-partitioning problem. Random Structures and Algorithms 30 (4) 505-531, (2007). MR2326156

[10] A. Bovier, I. Kurkova. Local energy statistics in disordered systems : a proof of the local REM conjecture. Commun. Math. Phys. 263 513-533 (2006). MR2207653

[11] A. Bovier, I. Kurkova. A tomography of the GREM : beyond the REM conjecture. Commun. Math. Phys. 263 535-552 (2006). MR2207654

[12] F. Comets, T. Shiga, N. Yoshida. Probabilistic analysis of directed polymers in a random environment: a review. Advanced Studies in Pure Mathematics 39 (2004), Stochastic Analysis on Large Scale Interacting Systems, 115-142. MR2073332

[13] B. Derrida. Random-Energy model : an exactly solvable model of disordered systems. Phys. Rev. B (3) 24(5) 2613-2626(1981). MR0627810

[14] B. Derrida. A generalisaton of the random energy model that incldes correlations betwen energies. Jounal Phys. Lett. 46, 401-407(1985).

[15] P. Erdos, S.J. Taylor, Some problems concerning the stucture of random walk paths. Acta Math. Acad. Sci. Hung. 11, 137-162 (1960). MR0121870

[16] W. Feller. An Introduction to Probability Theory and its Applications. Volume I. Third edition. John Wiley and Sons, (1970). MR0228020

[17] D.A. Huse, C.L. Henley. Pinning and roughening of domain wall in Ising systems due to random impurities, Phys. Rev. Lett. 54 2708-2711 (1985)

[18] J.Z. Imbrie, T. Spencer. Diffusion of directed polymer in a random environment. J. Stat. Phys. 52 (3)-(4), 609-625 (1988). MR0968950

[19] H. Krug, H. Spohn. Kinetic roughening of growing interfaces. In: Solids Far from Equilibrium C. Godrèche ed., Cambridge University Press (1991).

[20] S. Mertens. Phase transition in the number partitioning problem. Phys. Rev. Lett. 81(20), 4281-4284 (1998). MR1653530

[21] S. Mertens. A physicist's approach to number partitioning. Theoret. Comput. Sci. 265(1-2), 79-108, (2001). MR1848213 